\title{
Asociación entre sarcopenia medida con el índice del psoas por tomografía abdominal y complicaciones posoperatorias tempranas en pacientes con cáncer gástrico
}

\author{
Association between sarcopenia measured with the psoas index by \\ abdominal tomography and early postoperative complications in patients \\ with gastric cancer
}

Jaime Andrés Marín-Ordoñez $\mathbb{D}^{\mathbb{D}}$, Juan Carlos Osorno-Villegas² $\mathbb{D}$, Carlos Fernando Fuentes-Díaz ${ }^{3} \mathbb{D}$

1 Médico, especialista en Cirugía general, Hospital Militar Central. Dirección general de sanidad militar, Barranquilla, Colombia.
2 Médico, residente de Cirugía general, Universidad Militar Nueva Granada, Hospital Militar Central, Bogotá D.C., Colombia.
3 Médico, especialista en Cirugía general, Hospital Militar Central, Bogotá D.C., Colombia.

\section{Resumen}

Introducción. En Colombia el cáncer gástrico representa un problema de salud pública teniendo en cuenta su alta incidencia y sus elevadas tasas de mortalidad. Cerca del $15 \%$ de los pacientes sufren una pérdida significativa de peso, lo que se asocia con un incremento en la morbilidad y mortalidad.

Método. Se realizó un estudio de corte transversal, con el objetivo de determinar la presencia de sarcopenia a través de la medición del índice del psoas por tomografía computarizada y su asociación con morbimortalidad postoperatoria temprana en pacientes con cáncer gástrico. Se incluyeron los pacientes con cáncer gástrico admitidos entre el $1^{\circ}$ de enero de 2014 y el 31 de agosto de 2019 en el Hospital Militar Central, en Bogotá, D.C., Colombia. Se hizo un análisis descriptivo, un análisis bivariado y un análisis de regresión logística univariado para determinar la asociación de sarcopenia y complicaciones a 30 días. Todos los análisis fueron realizados en $\mathrm{R}^{\circledR}$.

Resultados. Se estudiaron 70 pacientes, encontrando una frecuencia de sarcopenia de 54,3\% (n=38), edad media de 69 años (RIC 54 - 74), mayor proporción de hombres 68,6 \% (n=48), siendo menor en el grupo de sarcopenia $55,3 \%$ (n=21), índice de psoas de 0,63 mm (RIC 0,55 - 0,7), mortalidad 2,9 \% ( $\mathrm{n}=2$ ) y asociación de sarcopenia con desenlaces a 30 días (OR 1,2; $\mathrm{IC}_{95 \%} 0,59$ - 2,4).

Fecha de recibido: 15/09/2020 - Fecha de aceptación: 11/12/2020 - Fecha de publicación en línea: 22/07/2021

Correspondencia: Jaime Andrés Marín Ordoñez, Carrera 68 \# 79-00, Barranquilla, Colombia. Teléfono: 3023458035

Correo electrónico: jaime112483@gmail.com

Citar como: Marín-Ordoñez JA, Osorno-Villegas JC, Fuentes-Díaz CF. Asociación entre sarcopenia medida con el índice del psoas por tomografía abdominal y complicaciones posoperatorias tempranas en pacientes con cáncer gástrico. Rev Colomb Cir. 2021;36: 647-56. https://doi.org/10.30944/20117582.850

Este es un artículo de acceso abierto bajo una Licencia Creative Commons - BY-NC-ND https://creativecommons.org/licenses/by-ncnd/4.0/deed.es 
Discusión. Se encontraron resultados similares a los informados en la literatura mundial, con una mortalidad inferior al 3 \%. En este estudio, la sarcopenia no se asoció con la aparición de complicaciones a 30 días.

Palabras clave: cáncer gástrico; medición; índice; psoas; tomografía; mortalidad.

\begin{abstract}
Introduction. Gastric cancer represents a public health problem in Colombia considering its high incidence and high mortality rates. About $15 \%$ of patients suffer a significant weight loss, which is associated with an increase in morbidity and mortality.
\end{abstract}

Method. A cross-sectional study was carried out in order to determine the presence of sarcopenia by measuring the psoas index by computed tomography and its association with early postoperative morbidity and mortality in patients with gastric cancer. Gastric cancer patients admitted between January 1, 2014 and August 31, 2019 at Hospital Militar Central, in Bogotá, D.C., Colombia were included. Descriptive analysis, bivariate analysis, and univariate logistic regression analysis were performed to determine the association of sarcopenia and complications at 30 days. All analyzes were performed in $\mathrm{R}^{\circledR}$.

Results. Seventy patients were studied, finding a frequency of sarcopenia of $54.3 \%$ (n=38), mean age of 69 years (IQR 54-74), higher proportion of men 68.6\% ( $n=48)$, being lower in sarcopenia group 55.3\% ( $\mathrm{n}=21)$, psoas index of $0.63 \mathrm{~mm}$ (IQR 0.55 - 0.7), mortality $2.9 \%(\mathrm{n}=2)$ and no association of sarcopenia with outcomes a 30 days (OR $1.2 ; 95 \%$ CI 0.59 - 2.4).

Discussion. Similar results were found to those reported in the world literature, with a mortality of less than $3 \%$. In this study, sarcopenia was not associated with the development of complications at 30 days.

Keywords: gastric cancer; measurement; index; psoas; tomography; mortality.

\section{Introducción}

A nivel mundial, el cáncer gástrico corresponde al quinto más frecuente, precedido del cáncer de pulmón, seno, colon y recto, y próstata. En el año 2012 se presentaron 952.000 casos nuevos y fue la causa de 738.000 muertes, constituyéndose en la tercera causa de muerte por cáncer en el mundo ${ }^{1}$.

De acuerdo con los estudios realizadas por el Instituto Nacional de Cancerología, en el 2006 se presentaron 7515 casos nuevos en Colombia, con una tasa estimada de 26,5 para hombres y de 15,4 para mujeres, tasas de incidencia similares a las de Costa Rica, Chile, Japón, Corea y China. En Colombia, corresponde a la primera causa de muerte por cáncer en hombres y a la tercera en mujeres ${ }^{2}$. Teniendo en cuenta la alta incidencia de cáncer gástrico, así como su mortalidad, este tumor representa un verdadero problema de salud pública para el país.
La estadificación con tomografía computarizada (TC) se debe realizar en todos los pacientes previo al procedimiento quirúrgico, para evaluar la extensión de la enfermedad, la presencia de metástasis y la invasión loco regional ${ }^{3}$. La cirugía continúa siendo el tratamiento primario para los pacientes con cáncer gástrico en estadio temprano. La resección completa con márgenes adecuados (4 cm o más) es considerada como el patrón de oro del tratamiento, mientras que el tipo de resección (subtotal vs. gastrectomía total) y la extensión de la disección linfática son aún tema de discusión ${ }^{3}$.

La sarcopenia relacionada solo con la edad se define como "primaria", mientras que en la sarcopenia "secundaria" se identifican una o más causas, como enfermedad inflamatoria, malignidad o desnutrición. El cáncer es posiblemente la afección patológica más notable que produce 
atrofia muscular, especialmente en pacientes de edad avanzada ${ }^{4}$. En los pacientes oncológicos, la sarcopenia es un fenómeno clínico que ha tomado importancia en los últimos años, y que denota una reducción en el volumen del músculo esquelético relacionado con pérdida de la función física, discapacidad, infecciones nosocomiales, mala respuesta a la quimioterapia y peor pronóstico ${ }^{4}$.

El objetivo de este estudio fue determinar la presencia de sarcopenia evaluada a través de la medición del índice del psoas por tomografía computarizada y su asociación con morbimortalidad postoperatoria temprana en pacientes con cáncer gástrico.

\section{Métodos}

Se realizó un estudio observacional de corte transversal, entre el $1^{\circ}$ de enero de 2014 y el 31 de agosto de 2019. Se incluyeron los pacientes con cáncer gástrico estadio I a III según el American Joint Committee on Cancer (AJCC), sometidos a gastrectomía (total o subtotal) en el Hospital Militar Central, en Bogotá, D.C., Colombia, con diagnóstico de adenocarcinoma gástrico confirmado por el estudio histopatológico y que contaran con tomografía de abdomen contrastada realizada como estudio de extensión dentro de los 30 días previos al tratamiento quirúrgico. Se excluyeron pacientes con mortalidad en el posoperatorio inmediato (primeras 24 horas) y aquellos sin datos disponibles en la historia clínica.

Se realizó un filtro de las historias clínicas con los códigos CIE-10 relacionados con el diagnóstico de cáncer gástrico, desde el C160 al C169 (Tumor maligno gástrico según localización anatómica), así como el D002 (Carcinoma in situ del estómago). Se analizaron los desenlaces en los primeros 30 días posteriores a la cirugía.

Las tomografías preoperatorias de abdomen de todos los pacientes fueron evaluadas en el formato Digital Imaging and Communications in Medicine (DICOM) y un médico radiólogo de la institución realizó el cálculo volumétrico del área de superficie de los músculos psoas en milímetros cuadrados $\left(\mathrm{mm}^{2}\right)$ y de manera bilateral, así como del cuerpo de la vertebra L4 que fue el punto de referencia de la localización axial. Las imágenes fueron evaluadas en el aplicativo Horos Project 64-bit versión 3.0 (LGPL 3,) para MAC iOs. La presencia de sarcopenia se trató como una variable categórica una vez fue calculado el índice de psoas cumpliendo el punto de corte establecido.

Se realizó el análisis descriptivo de las variables. Las variables categóricas se expresaron en proporciones y número absolutos; para las variables continuas se verificó el tipo de distribución con la prueba de Shapiro-Wilk, si los datos fueron de patrón de distribución normal, se expresaron con media y desviación estándar, y en caso contrario, se expresaron con mediana y rango intercuartílico.

La asociación de sarcopenia con morbimortalidad fue evaluada en primera instancia con un análisis bivariado, teniendo como variable dependiente las complicaciones a 30 días. Una vez obtenida esta evaluación se realizó un modelo de regresión logística univariado, donde la variable dependiente fue el desenlace de complicaciones a 30 días (dado el bajo número de pacientes y casos) y la variable independiente fue sarcopenia. Todos los análisis se realizaron en el software $\mathrm{R}^{\circledR}$, con licencia para la Universidad Militar Nueva Granada.

\section{Resultados}

Durante el periodo de estudio se identificaron 520 pacientes, de los cuales solo 38 cumplieron los requisitos para ser incluidos en el análisis (figura 1). La mediana de edad de la población se ubicó en la séptima década de la vida y en los pacientes del grupo de sarcopenia con una mediana de edad mayor (tabla 1). La mayor proporción de los pacientes fueron hombres, siendo esta proporción significativamente menor en el grupo de pacientes con sarcopenia. Los pacientes del grupo con sarcopenia presentaron un peso y talla menor que los del grupo de pacientes sin sarcopenia. El índice de masa corporal (IMC) de los pacientes se encontraba en el rango entre normalidad y sobrepeso; el índice de masa corporal de los pacientes con sarcopenia fue el más bajo, pero dentro del rango normal. 


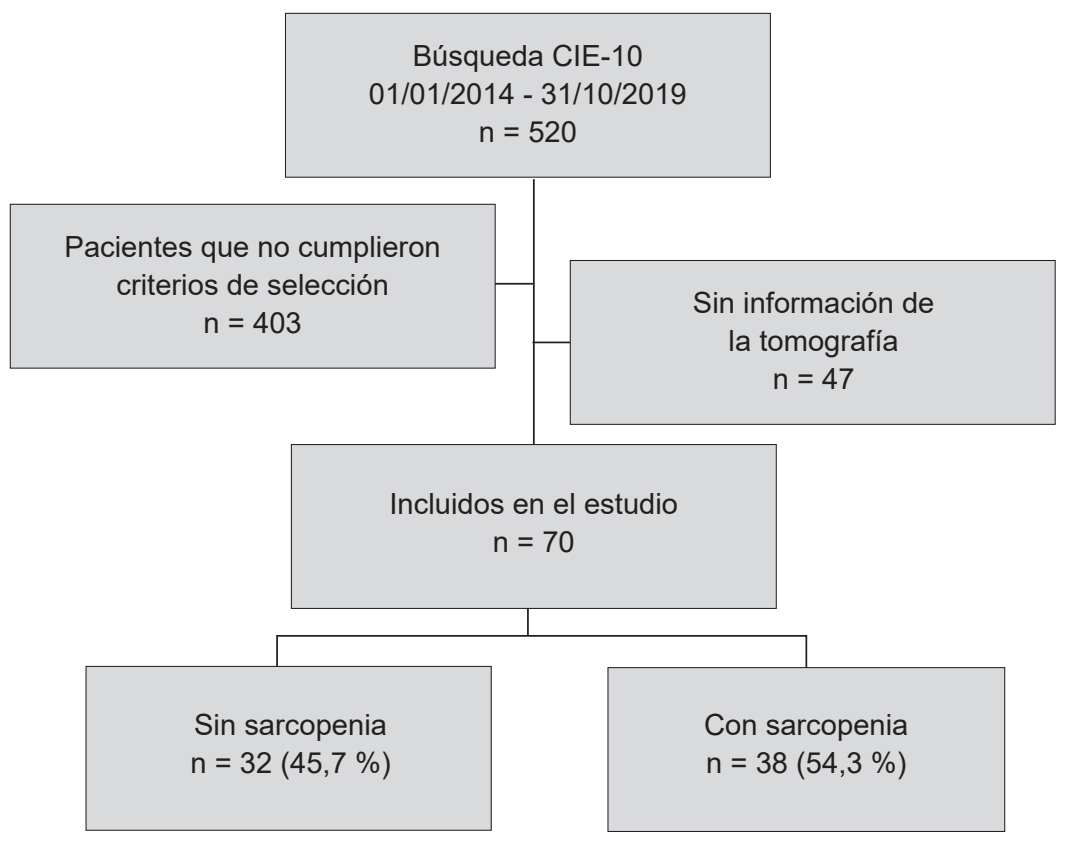

Figura 1. Flujograma de pacientes

Fuente: realizado por los investigadores

Tabla 1. Características de los pacientes incluidos en el estudio

\begin{tabular}{|c|c|c|c|c|}
\hline Variable & $\begin{array}{c}\text { Sin } \\
\text { sarcopenia } \\
n=32\end{array}$ & $\begin{array}{c}\text { Con } \\
\text { sarcopenia } \\
n=38\end{array}$ & $\begin{array}{l}\text { Total } \\
n=70\end{array}$ & $\mathbf{p}$ \\
\hline $\begin{array}{l}\text { Edad en años } \\
\text { mediana (RIC) }\end{array}$ & $63,5(45-72)$ & $72(60-78)$ & $69(54-74)$ & 0,21 \\
\hline Hombres, n (\%) & $27(84,4 \%)$ & $21(55,3 \%)$ & $48(68,6 \%)$ & 0,01 \\
\hline $\begin{array}{l}\text { Peso en } \mathrm{kg} \\
\text { mediana } \pm \mathrm{DE}\end{array}$ & $67,6 \pm 13,1$ & $60,8 \pm 11,6$ & $63,9 \pm 12,7$ & 0,09 \\
\hline $\begin{array}{l}\text { Talla en metros } \\
\text { mediana (RIC) }\end{array}$ & $1,64 \pm 0,06$ & $1,63 \pm 0,08$ & $1,64 \pm 0,07$ & 0,72 \\
\hline Índice de masa corporal mediana (RIC) & $24,7(21,7-26,6)$ & $22,8(19,4-26,0)$ & $23,7(20,4-26,4)$ & 0,34 \\
\hline Insuficiencia renal n (\%) & $2(6,2 \%)$ & $4(10,5 \%)$ & $6(8,6 \%)$ & 0,68 \\
\hline Diabetes mellitus $\mathrm{n}(\%)$ & 0 & $3(7,9 \%)$ & $3(4,3 \%)$ & 0,24 \\
\hline Hipertensión arterial n (\%) & $14(43,8 \%)$ & $16(42,1 \%)$ & $30(42,9 \%)$ & 0,99 \\
\hline \multicolumn{5}{|l|}{ Estadio de hipertensión arterial n (\%) } \\
\hline Estadio I & $4(28,6 \%)$ & $8(50 \%)$ & $12(40 \%)$ & 0,07 \\
\hline Estadio II & $10(71,4 \%)$ & $7(43,8 \%)$ & $17(56,7 \%)$ & 0,01 \\
\hline Estadio III & & $1(6,2 \%)$ & $1(3,3 \%)$ & 0,15 \\
\hline EPOC n (\%) & $1(3,1 \%)$ & $2(5,3 \%)$ & $3(4,3 \%)$ & 0,99 \\
\hline $\begin{array}{l}\text { Neumonía en el preoperatorio } \\
30 \text { días } \mathrm{n}(\%)\end{array}$ & 0 & $2(5,3 \%)$ & $2(2,9 \%)$ & 0,49 \\
\hline Tabaquismo n (\%) & $12(31,5 \%)$ & $9(23,7 \%)$ & $21(30 \%)$ & 0,29 \\
\hline Soporte nutricional preoperatorio $\mathrm{n}(\%)$ & $4(12,5 \%)$ & $5(13,2 \%)$ & $9(12,9 \%)$ & 0,99 \\
\hline Albúmina sérica g/dl, mediana (RIC) & $3,1(2,9-3,6)$ & $3,1(2,8-3,5)$ & $3,1(2,8-3,6)$ & 0,68 \\
\hline
\end{tabular}

RIC: rango intercuartílico; n: número; kg: kilogramos; DE: desviación estándar

Fuente: realizado por los investigadores 
El antecedente médico más frecuente fue el de hipertensión arterial, seguido de tabaquismo; se observó una frecuencia relativa mayor de antecedentes en los pacientes del grupo con sarcopenia. La evaluación del estado nutricional mediante albumina demostró que los pacientes presentaban valores normales.

La evaluación realizada por tomografía para determinar la presencia de sarcopenia demostró que la mediana del área de volumen del psoas derecho del total de los pacientes fue de $1344 \mathrm{~mm}^{2}$ (RIC 1019-1911), el volumen en el grupo con sarcopenia fue de $1211 \mathrm{~mm}^{2}$ (RIC 901-1647), estadísticamente menor que en el grupo sin sarcopenia $1742 \mathrm{~mm}^{2}$ (RIC 1268-2172) ( $\left.\mathrm{p}=0,03\right)$. Otra de las mediciones realizada fue el área de volumen del psoas izquierdo, la mediana general fue de 1486 mm $^{2}$ (RIC 1123-2068). Los pacientes con sarcopenia presentaron un área menor $1283 \mathrm{~mm}^{2}$ (RIC 837-1525), que la de los pacientes sin sarcopenia $2092 \mathrm{~mm}^{2}$ (RIC 1500-2445), diferencia estadísticamente significativa $(\mathrm{p}=0,001)$.

La evaluación del área del volumen de L4 no tuvo diferencias entre los pacientes con sarcopenia con una mediana de $1967 \mathrm{~mm}^{2}$ (RIC 1362-2429) y sin sarcopenia con una mediana de $1693 \mathrm{~mm}^{2}$ (RIC 1231- 2229) ( $\left.\mathrm{p}=0,15\right)$. Finalmente, el índice de psoas tuvo una mediana menor en el grupo de pacientes con sarcopenia $(0,63 \mathrm{~mm}$;
RIC 0,55-0,7) en comparación a los pacientes sin sarcopenia (1,11 mm; RIC 0,97-1,3), diferencia que fue estadísticamente significativa $(\mathrm{p}=0,001)$.

Con relación a las características de la enfermedad neoplásica, la mayor proporción de pacientes, equivalente al 35,7 \% (25/70) tenían un tumor en estadio T3, seguido de T2 en el 24,3\% (17/70) y T4a en el 15,7 \% (11/70) de los pacientes. No hubo diferencias estadísticamente significativas entre los dos grupos con relación a la distribución de la localización del tumor primario. El $100 \%$ de los pacientes estaba libre de metástasis. El nivel ganglionar observado con mayor frecuencia fue N0 con el 35,7 \% (25/70), seguido de N1 con el $20 \%(14 / 70)$, N2 con el 18,6 \% (13/70) y N3 con el 17,1\% (12/70), y no hubo diferencias estadísticamente significativas entre los grupos respecto a la distribución de las proporciones del nivel de invasión ganglionar.

El estadio tumoral en el que se encontraban los pacientes en el momento del estudio fue IIIA en el 25,7 \% (18/70), seguido de IIIB en el $18,6 \%$ (13/70), y los estadios IIA y IIIC en el 14,3\% (10/70). El 11,4\% (8/70) de los pacientes se encontraba con quimioterapia neoadyuvante, $12,5 \%$ (4/32) para el grupo sin sarcopenia y $10,5 \%(4 / 38)$ para el grupo con sarcopenia, diferencia que no fue estadísticamente significativa $(p=0,9)$.

Tabla 2. Características operatorias de los pacientes del estudio

\begin{tabular}{|c|c|c|c|c|}
\hline Variable & $\begin{array}{c}\text { Sin sarcopenia } \\
n=32\end{array}$ & $\begin{array}{l}\text { Con sarcopenia } \\
n=38\end{array}$ & $\begin{array}{l}\text { Total } \\
n=70\end{array}$ & $\mathbf{p}$ \\
\hline \multicolumn{4}{|l|}{ Estadificación del riesgo anestésico ASA n (\%) } & 0,99 \\
\hline II & $15(45,9 \%)$ & $18(47,4 \%)$ & $33(47,1 \%)$ & \\
\hline III & $17(53,1 \%)$ & $20(52,6 \%)$ & $37(52,9 \%)$ & \\
\hline Tiempo quirúrgico en horas, media $\pm \mathrm{DE}$ & $3,3 \pm 0,9$ & $3,4 \pm 0,8$ & $3,4 \pm 0,9$ & 0,91 \\
\hline Sangrado transoperatorio ml, mediana (RIC) & $100(50-200)$ & $100(50-200)$ & $100(50-200)$ & 0,97 \\
\hline \multicolumn{5}{|l|}{ Tipo de resección n (\%) } \\
\hline $\begin{array}{l}\text { R0 Ausencia de tumor residual } \\
\text { microscópicamente }\end{array}$ & $32(100 \%)$ & $31(81,7 \%)$ & $60(85,7 \%)$ & 0,99 \\
\hline $\begin{array}{l}\text { R1 Presencia de tumor residual } \\
\text { microscópicamente }\end{array}$ & 0 & $1(2,6 \%)$ & $1(1,4 \%)$ & \\
\hline
\end{tabular}

ASA: Sociedad Americana de Anestesia; DE: desviación estándar; ml: mililitros; RIC=rango intercuartílico Fuente: realizado por los investigadores 
En la tabla 2 se muestran los resultados con relación a las características operatorias y los desenlaces postoperatorio. El riesgo anestésico observado con mayor frecuencia fue ASA III, en la mitad de los pacientes. El tiempo quirúrgico promedio fue de tres horas, el sangrado transoperatorio tuvo una mediana de $100 \mathrm{ml}$ y al $90 \%$ de los pacientes se les realizó una resección R0.

La mediana de estancia total fue de 12 días, con una mediana de estancia en UCI de tres días. El desenlace más frecuente fue la sepsis, seguido de insuficiencia renal aguda, aunque ninguna superó el $10 \%$ del total de pacientes; no se encontró presencia de trombosis venosa profunda o embolismo pulmonar en el grupo de pacientes.

De los desenlaces relacionados con la herida quirúrgica, el más frecuente fue la infección de la herida, siendo esta incisional superficial en todos los casos. Las complicaciones más frecuentes fueron las tipo II y III de la clasificación Clavien-Dindo, no hubo diferencias entre los grupos. Se realizó transfusión de productos sanguíneos en el 12,9\% $(9 / 70)$ de los pacientes, $15,8 \%(6 / 38)$ en el grupo de sarcopenia y 9,4\% (3/32) en el grupo sin sarcopenia, sin diferencias estadística significativa $(p=0,49)$. Ninguno de los pacientes recibió nutrición enteral temprana posoperatoria (tabla 3).

Se evaluó la asociación de sarcopenia con el desenlace de complicaciones a 30 días, obteniendo un OR 1,2 (IC $95 \%$ 0,59 - 2,4), aunque el estimador puntual sugiere riesgo, el intervalo de confianza es muy amplio, esto en relación con el insuficiente tamaño muestral para la asociación con complicaciones a 30 días.

Tabla 3. Complicaciones postoperatorias de los pacientes.

\begin{tabular}{|c|c|c|c|c|}
\hline Variable & $\begin{array}{c}\text { Sin } \\
\text { sarcopenia } \\
n=32\end{array}$ & $\begin{array}{c}\text { Con } \\
\text { sarcopenia } \\
n=38\end{array}$ & $\begin{array}{l}\text { Total } \\
n=70\end{array}$ & $\mathbf{p}$ \\
\hline Estancia total en días, mediana (RIC) & $12(11-17)$ & $12(10-21)$ & $12(11-21)$ & 0,95 \\
\hline Estancia en UCl, mediana (RIC) & $3(2-4)$ & $3(2-5)$ & $3(2-4)$ & 0,60 \\
\hline \multicolumn{5}{|l|}{ Desenlaces a 30 días del postoperatorio } \\
\hline Mortalidad n (\%) & $1(3,1 \%)$ & $1(2,6 \%)$ & $2(2,9 \%)$ & 0,99 \\
\hline Infección de vías urinarias n (\%) & 0 & $3(7,9 \%)$ & $3(4,3 \%)$ & 0,25 \\
\hline Insuficiencia renal aguda n (\%) & $1(3,1 \%)$ & $3(7,9 \%)$ & $4(5,7 \%)$ & 0,62 \\
\hline Sepsis n $(\%)$ & $3(9,4 \%)$ & $2(5,3 \%)$ & $5(7,1 \%)$ & 0,65 \\
\hline Absceso intraabdominal postoperatorio a 30 días $\mathrm{n}(\%)$ & $2(6,2 \%)$ & $3(7,9 \%)$ & $5(7,1 \%)$ & 0,99 \\
\hline Fuga de la anastomosis n (\%) & $1(3,1 \%)$ & 0 & $1(3,3 \%)$ & 0,46 \\
\hline Infección de herida quirúrgica n (\%) & $4(12,5 \%)$ & $5(13,2 \%)$ & $9(12,9 \%)$ & 0,85 \\
\hline Dehiscencia de fascia n (\%) & $1(3,1 \%)$ & 0 & $1(3,3 \%)$ & 0,46 \\
\hline Grado de complicación según Clavien-Dindo n (\%) & $10(31,2 \%)$ & $15(39,5 \%)$ & $25(35,7 \%)$ & 0,58 \\
\hline I & $1(10 \%)$ & 0 & $1(4 \%)$ & \\
\hline II & $6(60 \%)$ & $12(80 \%)$ & $18(72 \%)$ & \\
\hline III & $1(10 \%)$ & $2(13,3 \%)$ & $3(12 \%)$ & \\
\hline IV & $1(10 \%)$ & 0 & $1(4 \%)$ & \\
\hline $\mathrm{V}$ & $1(10 \%)$ & $1(6,7 \%)$ & $2(8 \%)$ & \\
\hline
\end{tabular}

RIC: rango intercuartílico; UCl: unidad de cuidados intensivos; n: número Fuente: realizado por los investigadores 


\section{Discusión}

La depleción nutricional y la pérdida involuntaria de peso es un hecho común en los pacientes con cáncer. La relevancia clínica de la sarcopenia se debe a que la baja calidad muscular lleva a la discapacidad física, lo que deteriora el proceso de recuperación postoperatoria ${ }^{4}$.

A pesar de las diferencias en los puntos de corte, la frecuencia de sarcopenia preoperatoria encontrada en este estudio fue de 54,3\%. Según un metaanálisis de 13 estudios, principalmente americanos, realizado por Sehn Y. et al, la frecuencia de la sarcopenia varió entre el 12,5 \% y el $57,7 \%{ }^{5}$, siendo el límite superior similar a lo observado en este estudio. Un metaanálisis realizado por Kamarajah SK. et al, que incluyó 39 estudios con 8402 pacientes informó una frecuencia entre el $7 \%$ y el $70 \%$, este rango más amplio y mayor con respecto al metaanálisis anterior se debió a las técnicas de evaluación y a los puntos de corte empleados para definir sarcopenia ${ }^{6}$.

Con respecto a las características preoperatorias, la edad media de los pacientes del estudio estuvo en la séptima década de la vida, similar a lo observado en los dos metaanálisis mencionados previamente, donde la edad promedio fue superior a 60 años. Esto puede explicarse por la historia natural de la enfermedad y el pico epidemiológico asociado con la presentación de este tumor ${ }^{5,6}$. La mediana de edad de los pacientes con sarcopenia fue mayor a la de los pacientes sin sarcopenia. En el trabajo de Kamarajah SK. et al., los pacientes con esta condición se encontraban principalmente en la octava y novena década de la vida, lo que puede estar asociado a su condición oncológica pero también a factores propios de la edad $^{6}$.

El índice de masa corporal de los pacientes del estudio estuvo en el rango de normalidad a sobrepeso, hallazgo similar a lo observado en el metaanálisis de Kamarajah SK., et al., sin embargo, como fue descrito por Meng N., et al. y Kim KM. et al., este índice generalmente se encontrará en rangos normales o límite inferior en pacientes con sarcopenia y su uso, fuera de ajustar los modelos de predicción para complicaciones, es limitado y no se correlaciona con desenlaces de mortalidad ${ }^{6-8}$.

Un estudio realizado por Varilla MA., et al., en pacientes oncológicos de Colombia, buscó evaluar el estado nutricional empleando el IMC. En dicho estudio el cáncer gástrico correspondió al $32 \%$ del total de la población analizada, el $60 \%$ se encontraban en estado de desnutrición al momento de la evaluación, situación que contrasta con lo observado en este estudio, en el que el IMC se encontraba en el rango "normal". Esto puede ser explicado, como sugieren sus autores, por los diferentes estados de la enfermedad observados en estos pacientes ${ }^{9}$.

Las comorbilidades preoperatorias descritas en este estudio fueron la hipertensión arterial, el tabaquismo y la insuficiencia renal, comorbilidades propias de la edad y que generan una carga adicional al estado proinflamatorio usual de la sarcopenia, hallazgos similares a los informados por Sehn Y., et al., y Kamarajah SK., et al. ${ }^{5,6}$. La evaluación de la presencia de sarcopenia en este estudio fue determinada mediante tomografía computarizada como parte de los estudios de extensión preoperatorios de estos pacientes. Como se ha descrito, este método diagnóstico es considerado la prueba de oro para la evaluación de la composición corporal, ya que no solo brinda información de la cantidad sino de la calidad del músculo ${ }^{10,11}$.

La rápida adquisición de las imágenes, su uso rutinario en el seguimiento y tratamiento del cáncer, y su fácil interpretación, lo convierten en uno de los estudios de primera opción en nuestro medio, en comparación con la resonancia magnética o la ultrasonografía muscular, métodos que requieren gran entrenamiento y equipo especializado, o con el uso de biomarcadores sanguíneos, como el potasio sérico y la creatinina, que son medidas poco precisas y con múltiples factores preanalíticos que pueden llevar a resultados equívocos en la evaluación de la sarcopenia ${ }^{11,12}$.

Se han empleado otras pruebas diagnósticas para la evaluación de sarcopenia, como la densitometría por absorción de rayos X (DXA), la impedancia bioeléctrica, y la dilución de creatinina, sin embargo, según lo descrito por Buehring B., et 
al., estos métodos, además de no estar disponibles en nuestro medio, no tienen buena correlación con la tomografía y no aseguran una evaluación precisa ${ }^{13}$.

Con relación a las características de la neoplasia, la mayoría de los pacientes correspondieron al estadio T3 y T2, similar a lo observado por Zhou CJ., et al., en un estudio de 240 pacientes, donde el $80 \%$ se encontraban en esta misma estadificación. En nuestro estudio ningún paciente presentó compromiso metastásico, situación que es diferente a lo descrito por Lee JS, donde más del $80 \%$ de los pacientes tenían metástasis. Esto puede estar explicado por los criterios de inclusión, pues en este estudio no se incluyeron pacientes con enfermedad metastásica, con el fin de evaluar el impacto directo de la sarcopenia, sin la influencia del pronóstico derivado del compromiso a distancia ${ }^{14,15}$.

La evaluación de las características operatorias como el riesgo anestésico y el tiempo quirúrgico fueron similares a las observadas por Zhou CJ., et al., con características tumorales similares para ambas poblaciones, sin embargo, la resección curativa fue cercana al $90 \%$ en nuestro estudio, lo que difiere de lo observado por Zhou CJ., et al. y Sierzega M., et al., con resecciones curativas inferiores al $70 \%{ }^{14,16}$.

En el estudio de Kuwada K., et al., el sangrado observado fue de $300 \mathrm{ml}$ para los pacientes con sarcopenia y de $170 \mathrm{ml}$ para los pacientes sin sarcopenia, cifras superiores a las encontradas en esta investigación. Este hallazgo es relevante, teniendo en cuenta que se espera más sangrado en estos pacientes por la friabilidad de los tejidos ${ }^{17}$. El menor sangrado en este estudio puede estar explicado por el uso de sellantes de fibrina y los protocolos quirúrgicos desarrollados por el equipo quirúrgico para la atención de los pacientes.

La estancia hospitalaria total y en la unidad de cuidado intensivo en este estudio fueron similares a la descritas por Zhou CJ., et al., Sierzega M., et al. y Kuwada K., con una mediana entre 10 -16 días para la estancia total y 3 - 7 días para la estancia en UCI ${ }^{14,16,17}$. La mortalidad en los pacientes con cáncer gástrico sometidos a tratamiento quirúrgi- co fue similar a la informada por Sierzega M., et al. y Sehn Y., et al., oscilando entre el 0,1 - 3 \% 5,6,14,16.

Las complicaciones más frecuentes fueron infección de vías urinarias e insuficiencia renal aguda, hallazgos similares a los observados por Zhou CJ.y Sierzega M., con proporciones que van del $3 \%$ al $10 \%{ }^{5,14}$. La presencia de complicaciones de la herida quirúrgica, como absceso e infección del sitio operatorio varió entre el $7 \%$-13 \%, similar a lo reportado por Zhou CJ. ${ }^{14}$. De acuerdo con la clasificación de Clavien-Dindo, en este estudio, una tercera parte de los pacientes presentaron complicaciones, proporción mayor al $10 \%$ para ambos grupos descrita por Zhou CJ., esto puede ser explicado porque los pacientes de este estudio tuvieron mayor frecuencia de comorbilidades asociadas ${ }^{14}$. Las más frecuentes fueron las tipo II y III, hallazgos similares a lo descrito por Zhou CJ. ${ }^{14}$.

No se encontró asociación entre sarcopenia y desenlaces de morbilidad a 30 días, aunque el estimador puntual sugiere riesgo, el intervalo de confianza es muy amplio, esto en relación con el insuficiente tamaño muestral. En los estudios de Zhou CJ., Sehn Y. y Kamarajah SK., la sarcopenia sí se asoció a malos desenlaces en pacientes con cáncer gástrico, resultados que contrastan con lo observado por Tegels JJ., quien no encontró que la sarcopenia se asociara a mortalidad o complicaciones en una población con carcinoma gástrico, sin embargo, aunque su población es similar a la de este estudio, no son claros los motivos de la no asociación en esta población ${ }^{5-6,14,18}$.

Los resultados de este estudio son pioneros en nuestro país en evaluar, no solo el índice de masa corporal como marcador nutricional en pacientes con cáncer, sino el índice del psoas por tomografía para la evaluación de la sarcopenia. Esta es una aproximación a un problema que genera una gran carga de enfermedad en este grupo de pacientes que de por si tienen una condición debilitante y a la que, sumada la sarcopenia, incrementa la morbilidad, lo que ensombrecen el pronóstico de los pacientes.

Las limitaciones del estudio son su naturaleza retrospectiva, el bajo número de pacientes y de eventos postoperatorios, que disminuyó el poder 
para encontrar diferencias entre los grupos y asociaciones significativas. Debido a que no se logró el tamaño de muestra esperado para el estudio, los resultados a la fecha se consideran parciales, por lo que la continuidad de este estudio, en cabeza del grupo de cirugía oncológica del Hospital Militar Central, permitirá determinar la verdadera fuerza de asociación de sarcopenia en los pacientes con cáncer gástrico y predecir la aparición de desenlaces a un año, como lo sugieren Huang DD. y Kawamura T. ${ }^{19,20}$.

Adicionalmente, la identificación rutinaria del diagnóstico de sarcopenia en los pacientes con cáncer gástrico permitirá implementar medias farmacológicas, como lo sugiere la International Conference on Frailtyand Sarcopenia Research Task Force, y medidas no farmacológicas, como la realización de ejercicio y el soporte nutricional preoperatorio, que disminuyan el impacto negativo de la sarcopenia en los desenlaces postoperatorios, como lo sugiere Yamamoto K. ${ }^{21,22}$.

Los desenlaces de este estudio pueden generalizarse a pacientes adultos con diagnóstico de cáncer gástrico en plan quirúrgico, pero cualquier inferencia a la población fuera de este contexto debe ser revisada con cautela.

\section{Conclusiones}

La frecuencia de sarcopenia en los pacientes con cáncer gástrico sometidos a cirugía en el Hospital Militar Central fue del 54,3 \%. La mayor proporción de pacientes fueron hombres en la séptima década de la vida. El estado nutricional por índice de masa corporal fue normal o sobrepeso.

La resección con intención curativa fue mayor al $90 \%$ en los pacientes del estudio y la mortalidad fue menor al $3 \%$,valores similares a lo reportado por diferentes experiencias alrededor del mundo. La sarcopenia no se asoció al desenlace de morbilidad a 30 días.

\section{Cumplimiento de normas éticas}

Consentimiento informado: Este estudio es una revisión de historias clínicas retrospectiva, y como tal, no hay necesidad de un consentimiento informado. Esta investigación fue aprobada por el comité de ética en investigación del Hospital Militar Central.
Conflictos de interés: Los autores declaran no tener ningún conflicto de interés durante la realización y la publicación del estudio.

Fuente de financiación: Financiado con recursos propios de los autores.

\section{Contribución de los autores:}

Concepción y diseño del estudio: Jaime Andrés MarínOrdoñez, Juan Carlos Osorno-Villegas, Carlos Fernando Fuentes-Díaz.

Concepción y diseño del estudio: Jaime Andrés MarínOrdoñez, Juan Carlos Osorno-Villegas, Carlos Fernando Fuentes-Díaz.

Adquisición de datos: Jaime Andrés Marín-Ordoñez, Juan Carlos Osorno-Villegas, Carlos Fernando Fuentes-Díaz.

Análisis e interpretación de datos: Jaime Andrés MarínOrdoñez, Juan Carlos Osorno-Villegas, Carlos Fernando Fuentes-Díaz.

Redacción del manuscrito: Jaime Andrés Marín-Ordoñez, Juan Carlos Osorno-Villegas, Carlos Fernando FuentesDíaz.

Revisión crítica: Jaime Andrés Marín-Ordoñez, Juan Carlos Osorno-Villegas, Carlos Fernando Fuentes-Díaz.

\section{Referencias}

1. Gómez-Zuleta M, Riveros-Vega J, Ruiz O, Concha A, Ángel Betancur D, Torres Amaya M, et al. Guía de práctica clínica para la prevención, diagnóstico y tratamiento del cáncer gástrico temprano - 2015. Rev Colomb Gastroenterol. 2015;30(Supl 1):34-42

2. Ayala-Acosta JC, Lotero-Gómez JD. Tamización de cáncer gástrico. Univ Med. 2013;54:209-22.

https://doi.org/10.11144/Javeriana.umed54-2.tcga

3. National Comprehensive Cancer Network (NCCN). Gastric Cancer. 2017. https://www.nccn.org/professionals/physician_gls/pdf/gastric_blocks.pdf.

4. Ryall JG, Schertzer JD, Lynch GS. Cellular and molecular mechanisms underlying age-related skeletal muscle wasting and weakness. Biogerontology. 2008;9:213-28. https://doi.org/10.1007/s10522-008-9131-0

5. Shen Y, Hao Q, Zhou J, Dong B. The impact of frailty and sarcopenia on postoperative outcomes in older patients undergoing gastrectomy surgery: a systematic review and meta-analysis. BMC Geriatrics. 2017;17:188. https://doi.org/10.1186/s12877-017-0569-2

6. Kamarajah SK, Bundred J, Tan BHL. Body composition assessment and sarcopenia in patients with gastric cancer: a systematic review and meta-analysis. Gastric Cancer. 2019;22:10-22. https://doi.org/10.1007/s10120-018-0882-2 
7. Meng NH, Li CI, Liu CS, Lin WY, Lin CH, Chang CK, et al. Sarcopenia defined by combining height- and weight-adjusted skeletal muscle indices is closely associated with poor physical performance. J Aging Phys Act. 2015;23:597-606 https://doi.org/10.1123/japa.2014-0036

8. Kim KM, Jang HC, Lim S. Differences among skeletal muscle mass indices derived from height-, weight-, and body mass index-adjusted models in assessing sarcopenia. Korean J Intern Med. 2016;31:643-50. https://doi.org/10.3904/kjim.2016.015

9. Varilla MA, Restrepo MA, Pinzon OL, Valoyes E. Estado Nutricional de pacientes con neoplasias del tracto gastrointestinal. Nutr Clín Diet Hosp. 2017;37:139-146.

10. Tosato M, Marzetti E, Cesari M, Savera G, Miller RR, Bernabei R, et al. Measurement of muscle mass in sarcopenia: from imaging to biochemical markers. Aging Clin Exp Res. 2017;29:19-27. https://doi.org/10.1007/s40520-016-0717-0

11. Sergi G, Trevisan C, Veronese N, Lucato P, Manzato E. Imaging of sarcopenia. Eur J Radiol. 2016;85:1519-24. https://doi.org/10.1016/j.ejrad.2016.04.009

12. Ní ÉB, Daly LE, Power DG, Cushen SJ, MacEneaney P, Ryan AM. Computed tomography diagnosed cachexia and sarcopenia in 725 oncology patients: is nutritional screening capturing hidden malnutrition? J Cachexia Sarcopenia Muscle. 2018;9:295-305. https://doi.org/10.1002/jcsm.12258

13. Buehring B, Siglinsky E, Krueger D, Evans W, Hellerstein M, Yamada Y, et al. Comparison of muscle/lean mass measurement methods: correlation with functional and biochemical testing. Osteoporos Int. 2018;29:675-83. https://doi.org/10.1007/s00198-017-4315-6

14. Zhou CJ, Zhang FM, Zhang FY, Yu Z, Chen XL, Shen X, et al. Sarcopenia: a new predictor of postoperative complications for elderly gastric cancer patients who underwent radical gastrectomy. J Surg Res. 2017;211:137-46. https://doi.org/10.1016/j.jss.2016.12.014

15. Lee JS, Kim YS, Kim EY, Jin W. Prognostic significance of CT-determined sarcopenia in patients with advanced gastric cancer. PLoS ONE. 2018;13: e0202700. https://doi.org/10.1371/journal.pone.0202700
16. Sierzega M, Chrzan R, Wiktorowicz M, Kolodziejczyk P, Richter P. Prognostic and predictive implications of sarcopenia in Western patients undergoing gastric resections for carcinoma of the stomach. J Surg Oncol. 2019;120:473-82. https://doi.org/10.1002/jso.25509

17. Kuwada K, Kuroda S, Kikuchi S, Yoshida R, Nishizaki M, Kagawa S, et al. Sarcopenia and comorbidity in gastric cancer surgery as a useful combined factor to predict eventual death from other causes. Ann Surg Oncol. 2018;25:1160-6. https://doi.org/10.1245/s10434-018-6354-4

18. Tegels JJ, van Vugt JL, Reisinger KW, Hulsewé KW, Hoofwijk AG, Derikx JP, et al. Sarcopenia is highly prevalent in patients undergoing surgery for gastric cancer but not associated with worse outcomes.J Surg Oncol. 2015;112:403-7. https://doi.org/10.1002/jso.24015

19. Huang DD, Chen XX, Chen XY, Wang SL, Shen X, Chen XL, et al. Sarcopenia predicts 1-year mortality in elderly patients undergoing curative gastrectomy for gastric cancer: a prospective study. J Cancer Res Clin Oncol. 2016;142:2347-56. https://doi.org/10.1007/s00432-016-2230-4

20. Kawamura T, Makuuchi R, Tokunaga M, Tanizawa Y, Bando E, Yasui H, et al. Long-term outcomes of gastric cancer patients with preoperative sarcopenia. Ann Surg Oncol. 2018;25:1625-32. https://doi.org/10.1245/s10434-018-6452-3

21. Yamamoto K, Nagatsuma Y, Fukuda Y, Hirao M, Nishikawa K, Miyamoto A, et al. Effectiveness of a preoperative exercise and nutritional support program for elderly sarcopenic patients with gastric cancer. Gastric Cancer. 2017;20:913-8. https://doi.org/10.1007/s10120-016-0683-4

22. Vellas B, Fielding R, Bhasin S, Cerreta F, Goodpaster B, Guralnik JM, et al; International conference on frailtyand sarcopenia research task force. sarcopenia trials in specific diseases: report by the international conference on frailty and sarcopenia research task force. J Frailty Aging. 2016;5:194-200. 